\title{
New protein targets for treating liver cancer tied to hepatitis B
}

Hongjuan You

Dongchen Yuan

Yanwei Bi

Ning Zhang

Qi Li

Tao Tu

Xiao Wei

Qi Lian

Tong Yu

Delong Kong

Xiaoying Yang

Xiangye Liu

Xiaomei Liu

Fanyun Kong

Kuiyang Zheng

Renxian Tang

\section{Video Byte}

Keywords: Cell Communication and Signaling, hepatocellular carcinoma, HBX, vimentin, LASP1, epithelial mesenchymal transition, EMT, hepatitis b virus, Hepatitis $B$ virus $\mathrm{X}$ protein

Posted Date: October 15th, 2021

DOI: https://doi.org/10.21203/rs.3.rs-969886/v1

License: (c) (i) This work is licensed under a Creative Commons Attribution 4.0 International License.

Read Full License 


\section{Abstract}

Hepatocellular carcinoma is a significant cause of cancer-related death worldwide. This cancer is often tied to chronic infection by the hepatitis B virus (HBV). The pathway from viral infection to cancer is known to involve epithelial-mesenchymal transition (EMT) of cancerous cells, but the molecular details remain unclear. To gain a better understanding, researchers explored the relationship between two proteins: HBX, a hepatitis B-related protein reported to trigger EMT and vimentin, a structural protein that facilitates the EMT process. HBX-expressing cells and HBV-related tissue showed increased expression of the EMT markers vimentin, E-cadherin, and $\beta$-catenin. In addition, HBX-expressing cells showed greater proliferation and migration efficiency and larger tumor size in mice. Further analysis found that HBX was linked to vimentin via LASP1 and that multiple signal pathways are implicated. Understanding this connection between HBX and vimentin in hepatoma cells is crucial, as the two proteins could serve as potential targets of hepatocellular carcinoma treatment. 